\title{
Medical Image Denoising Techniques against Hazardous Noises: An IQA Metrics Based Comparative Analysis
}

\author{
Shakil Mahmud Boby \\ Department of EEE, Noakhali Science and Technology University, Noakhali-3814, Bangladesh \\ Email: shakilmahmud.eee97@gmail.com

\begin{abstract}
Shaela Sharmin
Department of EEE, Ahsanullah University of Science and Technology, Dhaka-1208, Bangladesh
\end{abstract} \\ Email: shaela.ritu@gmail.com
}

Received: 10 July 2020; Accepted: 15 September 2020; Published: 08 April 2021

\begin{abstract}
Medical imaging has become a vital part of the early detection, diagnosis, and treatment of many diseases. That's why image denoising is considered as a crucial pre-processing step in medical imaging to restore the original image from its noisy circumstance without losing image features, such as edges, corners, and other sharp structures. Ultrasound (US), Computed Tomography (CT), and Magnetic Resonance (MR) are the most widely used medical imaging techniques that are often corrupted by hazardous noises, namely, speckle, salt and pepper, Poisson, and Gaussian. To remove noises from medical images, researchers have proposed several denoising methods. Each method has its assumptions, merits, and demerits. In this paper, a detailed comparative analysis of different denoising filtering techniques, for example, median, Wiener, mean, hybrid median, Gaussian, bilateral, non-local means, and anisotropic diffusion are performed based on four widely-used image quality assessment (IQA) metrics, such as Root Mean Squared Error (RMSE), Peak Signal to Noise Ratio (PSNR), Mean Absolute Error (MAE), and Structural Similarity Index (SSIM). The results obtained in this present work reveal that Gaussian, median, anisotropic diffusion, and nonlocal means filtering methods perform extraordinarily to denoise speckle, salt and pepper, Poisson, and Gaussian noises, respectively, from all US, CT, and MR images.
\end{abstract}

Index Terms: Medical imaging, Image denoising techniques, US, CT, MR, Speckle, Salt and Pepper, Poisson, Gaussian, IQA metrics.

\section{Introduction}

Image denoising, one of the toughest challenges in image processing, refers to the recovery and quality enhancement of a digital image that has been tainted by noise during its acquisition, recording, or transmission [1]. It is an unavoidable function to eliminate or at least reduce the noise level from images. Even a small amount of noise is harmful when high accuracy is mandatory, like in the case of medical sector.

Medical images passed from Ultrasound (US) imaging, Computed Tomography (CT) imaging, and Magnetic Resonance Imaging (MRI) is the most widely used tools for diagnosis due to their non-invasive, harmless, and accurate nature [2]. Nevertheless, the imaging acquisition and systems may bring in unwanted noises, most commonly, speckle noise, salt and pepper noise, Poisson noise, and Gaussian noise [3-5]. In consequence, these deteriorate the visual evaluations, i.e., image resolutions and contrasts very severely that make it difficult to distinguish normal and pathological tissues in diagnostic examinations [6]. Thus, to achieve the best diagnosis, it is the mandatory preprocessing step to denoise medical images without altering edges, losing essential features of the images, and destroying anatomical details $[7,8]$.

US utilizes the echoes transmitted back to the transducer from any body tissue when sending an ultrasonic wave to it. Using each echo's return time and speed of sound, the ultrasonic scanner calculates the distance between the body tissue and the transducer probe and finally transformed it into a digital image [9]. Grievously, ultrasound images experience different noises due to the loss of proper contact or air gap between the transducer probe and body [3, 10].

$\mathrm{CT}$ is a technique utilizing tomography to produce a three-dimensional (3D) image of the internals of humans or any objects from an extensive series of 2D X-ray images taken around a single axis of rotation. In X-ray technology, radiation is exposed to the object, and reflection is made to be produced on 2D projection radiographs [4]. The number 
of X-ray photons coming at the detector specifies the noise in the CT image. The reconstruction kernel and electronic noise of the detector system provide noisier images [11].

Unlike X-ray radiography, MRI produces detailed anatomical images without the damaging radiation. Here, the object experiences a strong magnetic field that aligns the protons in the body according to that field. The electronic interferences in the receiver circuits, radiofrequency emissions due to thermal motion of the ions in patient body, and the measurement chain of MRI scanners mainly result in noises in MRI images [4, 5].

Spatial filtering and multiscale methods are the two conventional approaches for eliminating or reducing noises from different medical images. The spatial filtering is used immediately after the data is stored while at the data acquisition stage, the multiscale method is applied. To fulfill the requirements, i.e., conserving radiometric data, edge information, and spatial resolution, any of those methods can be applied [12]. Frost filtering [13], Lee filtering [14], Kuan filtering [15], mean filtering [16], Wiener filtering [17], and median filtering [18] are the techniques under spatial filtering domain. To reduce medical noises, local statistical properties are used in this particular filtering domain. The special Frost, Lee, and Kuan filtering methods were primarily investigated by the synthetic aperture radar (SAR) research community. Though later they become useful to denoise the medical images, they face some complications. These filtering methods are only feasible in a bounded field, and they are potentially used to remove only speckle noise [13-15]. On the contrary, multiscale filtering covers several methods that use the wavelet transform to hold the image signal irrespective of its frequency content [19, 20]. Another image denoising tool is the total variation (TV) model proposed by Rubin et al. [21] and the anisotropic diffusion model proposed by Perona and Malik [22]. Other widely used speckle noise reduction techniques are non-local means method [23], bilateral filtering method [24], and Gaussian filtering method [25]. Along with these, there are many other techniques and algorithms for removing noises from medical images [26, 27].

To appraise the functioning of each of the filtering methods, it is essential to have some metrics for image quality assessment (IQA). These metrics are grouped into two categories: full reference (FR) approach that uses a reference image and no-reference (NR) approach, which has no reference image to compare with the test image [28]. The root mean squared error (RMSE), peak signal to noise ratio (PSNR), mean absolute error (MAE) are the most widely used and also the most straightforward FR metrics [29]. But they are not always very perfect for discerning visual quality and also are not normalized in representation. That's why one of the normalized FR methods, namely, structural similarity index (SSIM), has become popular as IQA metrics, consequently [30].

All the image denoising filtering methods mentioned earlier have their particular merits and demerits [13-27]. Since noise-free medical imaging is a vital element of the healthcare world and an essential tool for physicians to assist with diagnostics, treatment, and prevention, it is essential to identify the best filtering methods for particular noise types. To best of our knowledge, nobody has reported the finest filtering methods yet for all possible hazardous noises experienced in different types of medical images in a single study. In the present study, we have introduced a comparative analysis of eight conventional filtering techniques (median, Wiener, mean, hybrid median, Gaussian, bilateral, non-local means, anisotropic diffusion) based on four primary IQA metrics (RMSE, PSNR, MAE, SSIM) against four ordinary noise types (speckle, salt and pepper, Poisson, Gaussian) in three different medical images (US, CT, MRI). The remainder of this paper is organized as follows. The materials and methods are illustrated in section II. Section III presents the results analysis and discussion. Ultimately, section IV concludes the entire work.

\section{Materials and Methods}

\section{A. Noise Models}

\section{1) Speckle Noise Model}

In medical imaging, speckle, one kind of granular interference is most likely deliberated as the source of noise. It masks small differences in the gray level that makes it an undesirable property of the images [7]. Speckle is mainly a form of multiplicative noise that is image dependent, complex to model, and hence difficult to reduce. Although speckle noise is defined as multiplicative noise, it can be converted into additive noise through log operation. The generalized model of the speckle noise can be represented as [31]

$$
N(x, y)=O(x, y) * m(x, y)+a(x, y)
$$

Here, $N(x, y)$ represents the observed noisy image, $O(x, y)$ is the original image, $m(x, y)$ is denoted as multiplicative noise component, and $a(x, y)$ is the additive component of the speckle noise. Here, $x$ and $y$ specify the pixel positions, i.e., axial and lateral indices of the image, respectively.

For medical imaging, only the multiplicative component is considered for calculation. Hence, the additive component of (1) can be neglected and can further be represented as

$$
N(x, y)=O(x, y) * m(x, y)
$$


After applying the logarithmic operation, (2) becomes

$$
\log _{e} N(x, y)=\log _{e} O(x, y)+\log _{e} m(x, y)
$$

Here (3) is the final model representation of speckle noise in terms of additive formation.

\section{2) Salt and Pepper Noise Model}

Salt and pepper noise is a non-continuous series of irregular spikes of short duration, which mainly generates due to the quick transition of imperfect switching. It may also be caused due to dead pixel, error in analog to digital conversion, or bit transmission error. Salt and pepper noise is also termed as impulse noise.

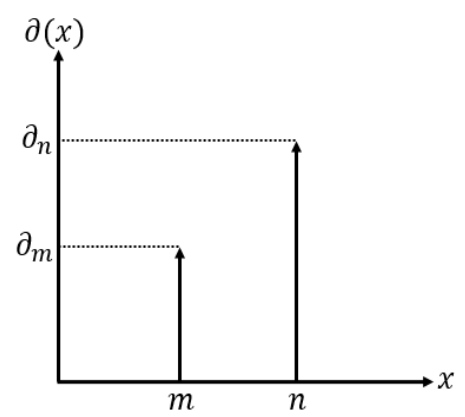

Fig. 1. The probability density function for salt and pepper noise.

This noise is generally produced due to the error in transmission of bit. That's why it can have only two possible values, i.e., $m$ and $n$. Usually, each of them has a probability of less than 0.1 . The minimum or the maximum value of the corrupted pixels is set possibly, and salt and pepper image become appeared [32]. Fig. 1 illustrates the function of the probability density for salt and pepper noise.

Salt and pepper noise can be individualized by using the following equation

$$
\partial(x)=\left\{\begin{array}{l}
\partial_{m} ; x=m \\
\partial_{n} ; x=n \\
0 ; \text { otherwise }
\end{array}\right.
$$

When $m>n$, then level of $m$ and $n$ become appeared as light dot and dark dot respectively and vice-versa.

\section{3) Poisson Noise Model}

When energy-carrying particles such as photons in an optical device or electrons in an electronic circuit are insufficient in amount to rise detectable statistical fluctuations in measurement, Poisson noise arises. It is an electronic noise whose magnitude depends on signal type and comprises the dominant source. The Poisson process is widely used to model statistically different engineering and science-based severe problems. The medical images corrupted by noises obey a Poisson law and are highly dependent on the underlying light intensity pattern being imaged. In the case of Poisson noise, the noise variance is proportional to the image pixels [33].

$$
E\left[\frac{z_{j}}{y_{j}}\right]=y_{j}=\operatorname{var}\left[\frac{z_{j}}{y_{j}}\right] ; j=1, \ldots \ldots, N
$$

Here, $z_{j}$ is the observed pixel values obtained through an image acquisition device, which is also considered as independent random Poisson variable. $y_{j}$ is the estimated underlying intensity value and considered as mean of $z_{j}$.

\section{4) Gaussian Noise Model}

Due to thermal atomic vibration and discrete radiation of objects, Gaussian noise is caused. It generally arises in detectors or amplifiers; that's why it is also an electronic noise. Since Gaussian noise badly affects the gray values in a digital image, its model is usually designed and characterized by a probability distribution function or normalized histogram with respect to gray values [34]. Fig. 2 shows the probability distribution function of Gaussian noise.

$$
P(z)=\frac{1}{\sqrt{2 \pi} \sigma} e^{-\frac{(z-\mu)^{2}}{2 \sigma^{2}}}
$$


Here, $z$ represents gray value, $\sigma$ denotes standard deviation, $\mu$ specifies mean value, and $\sigma^{2}$ indicates variance. Generally mathematical model of Gaussian noise represents the correct approximation of real-world scenarios. In this noise model, mean value and variance are 0 and 0.1 , respectively, and 256 gray levels exist in terms of its probability distribution function.

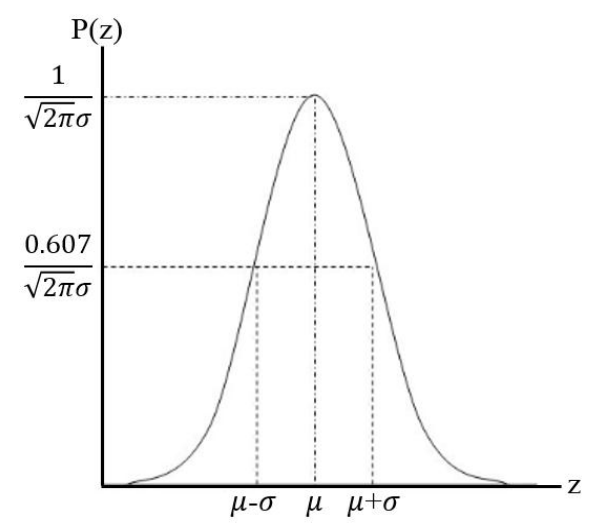

Fig. 2. The probability distribution function of Gaussian noise.

\section{B. Filtering Techniques}

\section{1) Median Filter}

In digital image processing, the median filter is a nonlinear filtering technique, which is also known as an orderstatistic filter. This filtering technique is prevalent for the reduction of impulse noise from an image or signal. It operates pixel by pixel and replaces the value of each cell with the neighboring by a median of the intensity levels with its mathematical accuracy. The median filter is applied over windows of size $5 \times 5$. Three different windows are used to calculate the output median by the median filter, namely, normal window, $\mathrm{x}$-shape window, and cross shape window. For improving the optical perception, median filters are easily suited. But repeated uses may damage the picture boundaries. The further restriction includes computational cost with complexity [18].

\section{2) Wiener Filter}

Wiener filter uses a linear time-invariant (LTI) filtering technique that can recover information from badly degraded or blurred images. The reduction of noise from the images is performed by considering a noiseless image as a reference. Wiener filter is based on the computation of local image variance, and the value of variance has a significant impact on the performance of this filter. With a small value of local variance, Wiener filter executes more smoothing. On the contrary, comparatively lesser smoothing is performed while local variance has a larger value [35]. Wiener filter is given by

$$
f(m, n)=\left\{\frac{H(m, n)^{*}}{H(m, n)^{2}+\left(\frac{S i(m, n)}{S f(m, n)}\right)}\right\} G(m, n)
$$

Where, $H(m, n)$ represents the degradation function, and its complex conjugate is denoted by $H(m, n)^{*}$. $G(m, n)$ denotes the degraded image, and $S f(m, n)$ and $\operatorname{Si}(m, n)$ indicate the power spectra of the original image and the noisy image, respectively.

\section{3) Mean Filter}

It is a basic filtering technique used to smoothen the image by adjusting the neighboring pixels. Convolution and kernels are the principal basis of this filtering method. Often a $3 \times 3$ square kernel is used, although larger kernels (e.g., $5 \times 5$ square) can be used for more smoothing. It destroys the pixel value if it does not match with the surrounding pixels [36]. 


\begin{tabular}{|c|c|c|}
\hline$\frac{1}{9}$ & $\frac{1}{9}$ & $\frac{1}{9}$ \\
\hline$\frac{1}{9}$ & $\frac{1}{9}$ & $\frac{1}{9}$ \\
\hline$\frac{1}{9}$ & $\frac{1}{9}$ & $\frac{1}{9}$ \\
\hline
\end{tabular}

Fig. 3. A $3 \times 3$ square kernel used for mean filtering.

\section{4) Hybrid Median Filter}

In modern days hybrid median filter plays a vital role in vision and image processing. It is a windowed filter of nonlinear class that can quickly dispel impulse noise while preserving the edges. If side by side comparison is made, hybrid filters show much better corner preserving characteristics than ordinary median filters. This filter is defined as [37]

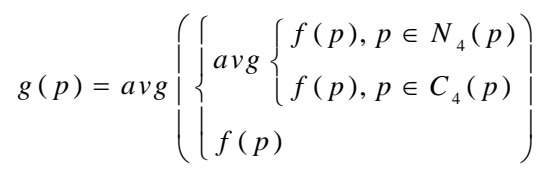

\section{5) Gaussian Filter}

Gaussian filter is a linear filter that is very effective for removing speckle noise in medical images. The characteristics of this filter are similar to that of the median filter, but the difference is that it uses different kernels with the bell-shaped distribution. The advantage of the Gaussian filter over the median filter is that it exhibits faster operation. Mathematically, the input signal is modified by convolution with a Gaussian function, which results in smoothing of the image. The 2D Gaussian distribution with standard deviation $\sigma$ for a pixel $(i, j)$ is given by [25]

$$
G(x)=\frac{1}{2 \pi \sigma^{2}} \exp \left(\frac{i^{2}+j^{2}}{2 \sigma^{2}}\right)
$$

As a matter of performance, the value of standard deviation controls the amount of blurring. If the value of the standard deviation is large, it makes a wide peak, which ultimately influences more significant blurring. Besides, the degree of blurring is also controlled by the size of the kernel. Usually, an odd number of pixels like $3 \times 3$ pixel, 5 x 5 pixel is used to square the kernel size. This is done to make the pixel being acted upon in the middle. With the increasing value of standard deviation, kernel size must increase to maintain the Gaussian nature of the filter.

\section{6) Bilateral Filter}

Bilateral filtering is a nonlinear filtering technique where smoothing an image with preserving edges is the main function, just like anisotropic diffusion. But the critical difference is that bilateral filtering is not iterative, and it consists of both range and domain filtering [24]. The bilateral filter is defined as

$$
B F(x)=k^{-1}(x) \int_{-\infty}^{\infty} \int_{-\infty}^{\infty} f(\xi) c(\xi, x) s(f(\xi), f(x)) d \xi
$$

The normalization term $k(x)$ can be expressed as

$$
k(x)=\int_{-\infty}^{\infty} \int_{-\infty}^{\infty} c(\xi, x) s(f(\xi), f(x)) d \xi
$$

Here, $f(x)$ represents the input image, $c(\xi, x)$ is the geometric closeness between the pixel and a near point $\xi$, and $s(f(\xi), f(x))$ denotes the photometric similarity between the pixels $x$ and $\xi$. 


\section{7) Non-Local Means (NLM) Filter}

Non-local means is an algorithm in image processing that is used for noise reduction. The Non-Local Means (NLM) filter was introduced by Buades et al. [23] where a mean of all pixels in the image are taken and weighted by how similar these pixels are to the target pixel. The expression for restored intensity of a pixel $x_{i}$ can be expressed as

$$
N L(v)\left(x_{i}\right)=\sum_{x_{j} \in \Omega^{\mathrm{dim}}} w\left(x_{i}, x_{j}\right) v\left(x_{j}\right)
$$

Here, $w\left(x_{i}, x_{j}\right)$ represents the weight given to $v\left(x_{j}\right)$ to reinstate the pixel $x_{j}$ and calculated as

$$
w\left(x_{i}, x_{j}\right)=\frac{1}{Z_{i}} \exp \left(-\frac{\left\|v\left(I_{i}\right)-v\left(I_{j}\right)\right\|_{2, a}^{2}}{h^{2}}\right)
$$

Where $I_{i}$ and $I_{j}$ are the intensities of local neighborhood centers on pixels $x_{i}$ and $x_{j}, Z_{i}$ indicates normalization constant, and $h$ is the degree of filtering, which controls the decay of the exponential function.

\section{8) Anisotropic Diffusion Filter}

Anisotropic diffusion, which is also known as Perona-Malik diffusion, is a technique that targets of reducing image noise without removing significant details that are essential for the interpretation of the image. This method was proposed by Perona and Malik, where reduction of image blurring, resolving of localization problem, enhancement of contrast, retaining of image edges as well as ultimate improvement of image quality are ensured by a partial differential equation (PDE) based nonlinear and space-variant filtering method [23]. This filtering method can be directly applied to the images to dispel noise. The diffusion process of the image can be expressed as [22]

$$
\left\{\begin{array}{l}
\frac{\partial i(x, y, t)}{\partial t}=\operatorname{div}[c(|\nabla i(x, y, t)|), \nabla i(x, y, t)] \\
i(x, y, t=0)=i_{0}(x, y)
\end{array}\right.
$$

Where, $\nabla$ and $d i v$ are the gradient and divergence operator respectively, $i_{0}(x, y)$ is the initial image and $c(|\nabla i(x, y, t)|)$ denotes the diffusion coefficient that can be represented by

$$
c(|\nabla i(x, y, t)|)=\exp \left(-\left(\frac{\|\nabla i(x, y, t)\|}{k}\right)^{2}\right)
$$

Here, $k$ is an edge magnitude parameter. Though narrated anisotropic diffusion filter exhibits better performance for additive noise, in case of multiplicative noise like speckle noise, its accomplishment is not up to the mark. Considering this problem, $\mathrm{Yu}$ and Acton have proposed an anisotropic diffusion filter, particularly for speckle retrenchment [38]. Here the same (14) is used where $c(\ldots . .$.$) is a function of the instantaneous coefficient of variation$ $q(x, y, t)$ and is given by the ratio of standard deviation to mean as

$$
q(x, y, t)=\frac{\operatorname{std}(i(x, y, t))}{\overline{i(x, y, t)}}
$$

The diffusion function $c(\ldots \ldots)$ is given by

$$
c\left(q(x, y, t), q_{0}(t)\right)=1+\frac{q^{2}(x, y, t)-q_{0}(t)}{q^{2}(x, y, t)\left(1+q_{0}^{2}(t)\right)}
$$

Here, $q_{0}(t)$ implies the speckle scale function. 


\section{Image Quality Assessment (IQA) Metrics}

\section{1) Root Mean Squared Error (RMSE)}

RMSE is a frequently used error measuring method that measures the difference between the actual value and any predicted value. The final value of RMSE is always non-negative and indicates an error magnitude. It is nothing but the square root of MSE. The lower value of RMSE exhibits better performance [30].

$$
R M S E=\frac{1}{\sqrt{U V}} \sum_{p=1}^{U} \sum_{q=1}^{V}[B(p, q)-A(p, q)]
$$

\section{2) Peak Signal to Noise Ratio (PSNR)}

PSNR can be defined as the ratio between the maximum possible signal power and distorted noise signal power. It is usually calculated in the decibel (dB) scale. PSNR is the most widely used quality assessment parameter to measure the quality of noisy reconstructed images. The signal is considered as the original data, and the noise is the error yielded by the compression or distortion. PSNR is expressed as [30]

$$
P S N R=10 \log _{10}\left(\frac{p e a k^{2} * U V}{\sum_{p=1}^{U} \sum_{q=1}^{V}[B(p, q)-A(p, q)]^{2}}\right)
$$

Here, peak denotes the maximum image data value that is 255 for an 8-bit unsigned data type. A higher value of PSNR indicates better performance of the filter.

\section{3) Mean Absolute Error (MAE)}

The average of absolute error between the original image, as reference, and the reconstructed image, as output is known as MAE. The lower value of MAE is considered better for any image analysis filter [29, 30]. The mean absolute error is given by

$$
M A E=\frac{1}{U V} \sum_{p=1}^{U} \sum_{q=1}^{V}|B(p, q)-A(p, q)|
$$

\section{4) Structural Similarity Index (SSIM)}

For measuring the similarity between original and reconstructed images, SSIM is often considered as a promising metric. It exhibits much better consistency with the qualitative appearance of the images. SSIM has a value between 0 and 1. When SSIM equals to 1, this indicates that images are structurally equal. SSIM is expressed by [29]

$$
\operatorname{SSIM}=\frac{\left(2 \mu_{p} \mu_{q}+C_{1}\right)\left(2 \sigma_{p q}+C_{2}\right)}{\left(\mu_{p}^{2}+\mu_{q}^{2}+C_{1}\right)\left(\sigma_{p}^{2}+\sigma_{q}^{2}+C_{2}\right)}
$$

Where $C_{1}$ and $C_{2}$ are two constants.

\section{Proposed Methodology}

Fig. 4 shows the flowchart of the proposed methodology for this research work.

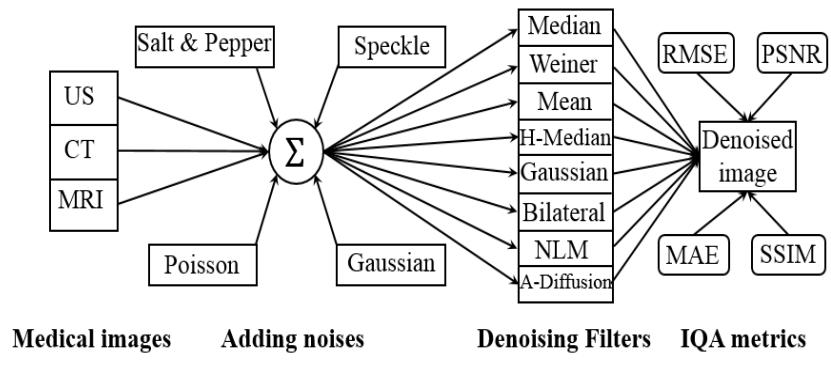

Fig. 4. Flowchart of the proposed methodology.

- Firstly, each of the medical images (US, CT, MRI) are intentionally made corrupted by speckle noise, salt and pepper noise, Poisson noise, and Gaussian noise. 
- Secondly, noisy medical images are denoised by all the eight filtering methods, namely, median, Weiner, mean, hybrid median (H-Median), Gaussian, bilateral, non-local means (NLM), and anisotropic diffusion (A-Diffusion), respectively.

- Finally, the performance of all the filtering techniques is compared based on RMSE, PSNR, MAE, and SSIM image quality assessment metrics.

\section{Results Analysis and Discussion}

In this section, the implementation of different denoising filtering techniques is carried out upon several real-time medical images. To make this research work more justifiable, here three different types of original gray ( $256 \times 256$ ) medical images, such as US image, CT image, and MRI image are taken into consideration, as shown in Fig. 5. Then the procedures of the proposed methodology are followed.

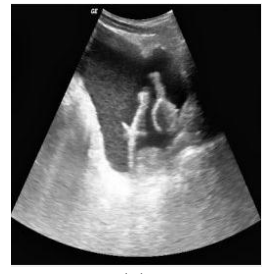

(a)

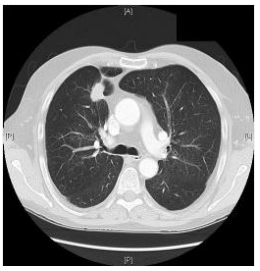

(b)

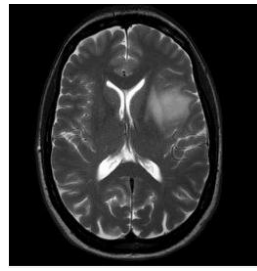

(c)

Fig. 5. Real-time 256 x 256 original medical images (a) US image, (b) CT image, and (c) MRI image.

(a)

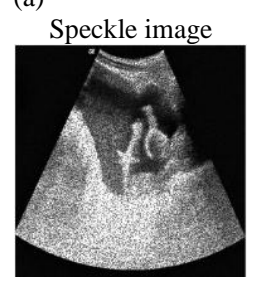

Mean

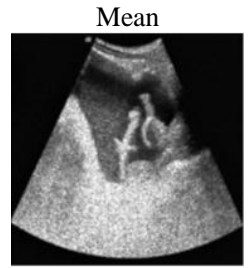

Bilateral

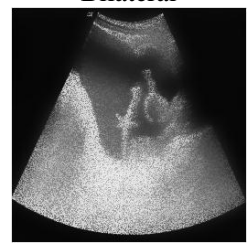

(c)

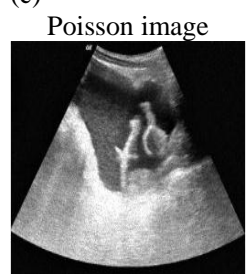

Mean

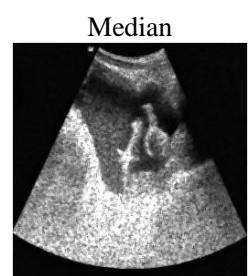

Hybrid Median

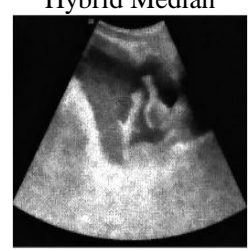

NLM

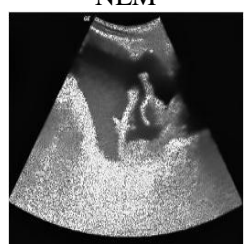

Median

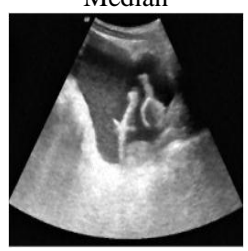

Hybrid Median

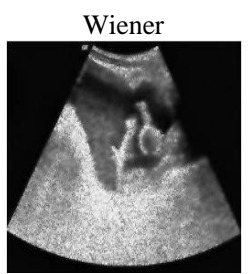

Gaussian

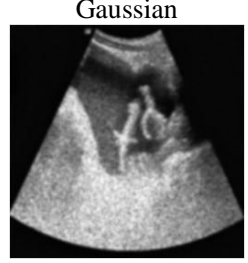

Anisotropic Diff.
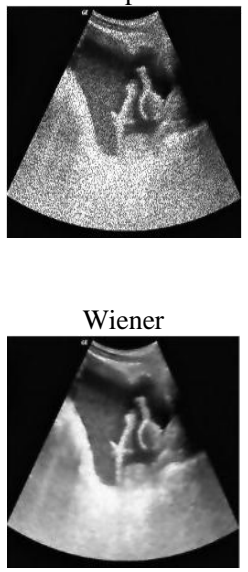

Gaussian (b)

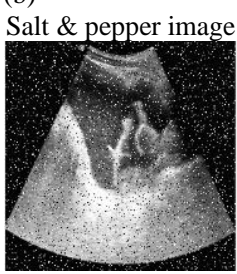

Mean

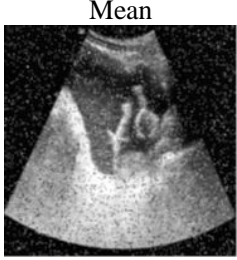

Bilateral

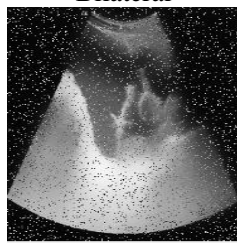

(d)

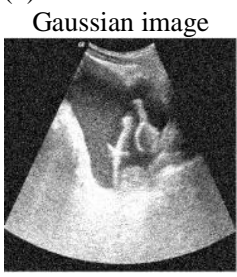

Mean

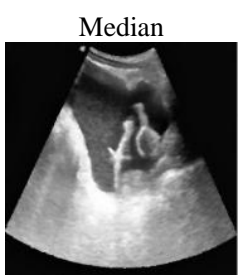

Hybrid Median

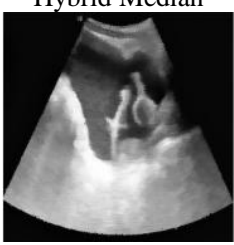

NLM

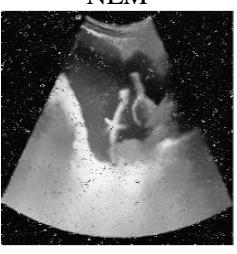

Median

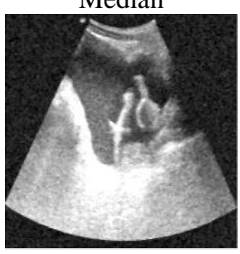

Hybrid Median

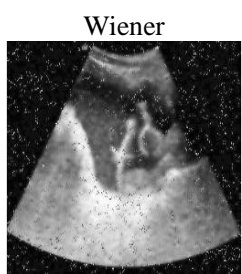

Gaussian

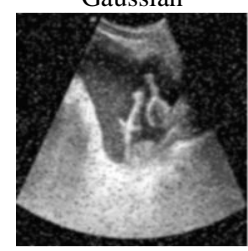

Anisotropic Diff.

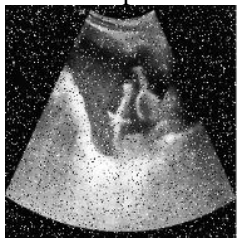

Wiener

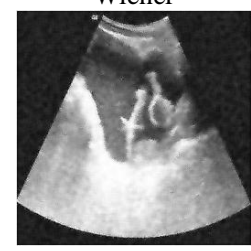

Gaussian 

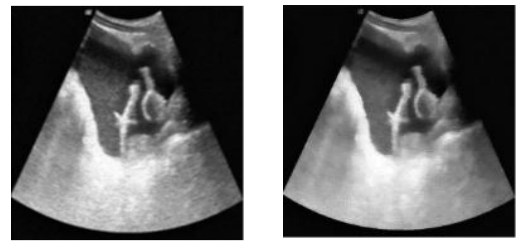

Bilateral
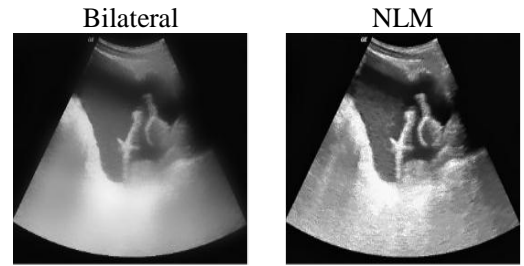

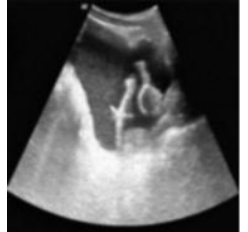

Anisotropic Diff.

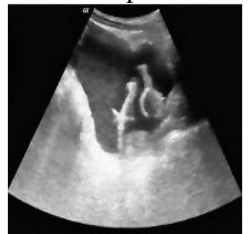

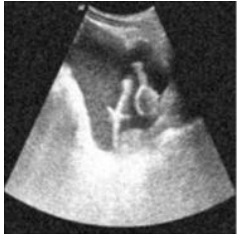

Bilateral

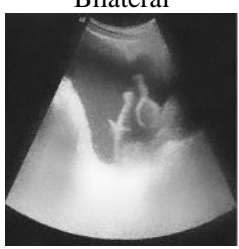

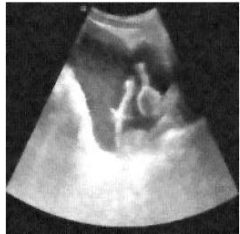

NLM

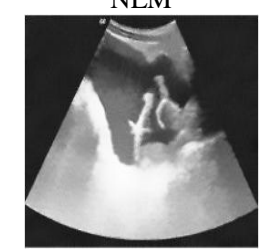

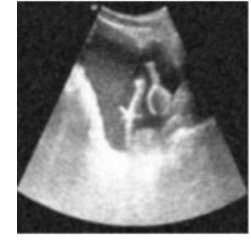

Anisotropic Diff.

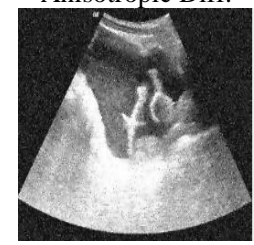

Fig. 6. Comparative visual quality analysis of US images, denoised by different filtering techniques: Median, Wiener, Mean, Hybrid Median, Gaussian, Bilateral, Non-Local Means, and Anisotropic Diffusion. Here, (a) different filtered images corrupted by speckle noise $($ variance = 0.1); (b) different filtered images corrupted by salt \& pepper noise (variance $=0.1)$; $(\mathrm{c})$ different filtered images corrupted by Poisson noise $($ variance $=0.1$ ); and (e) different filtered images corrupted by Gaussian noise (variance $=0.1$ ).

Table 1. Quantitative data values of RMSE, PSNR, MAE, and SSIM image quality assessment metrics of different filtering techniques corresponding to speckle noise, salt and pepper noise, Poisson noise, and Gaussian noise with variance $=0.1$ respectively for 2D gray $256 \mathrm{x} 256 \mathrm{US}$ image.

\begin{tabular}{|c|c|c|c|c|c|c|c|c|c|c|c|c|c|c|c|c|}
\hline Noise Types & \multicolumn{4}{|c|}{ Speckle $($ var. $=0.1)$} & \multicolumn{4}{|c|}{ Salt and Pepper $($ var. $=0.1)$} & \multicolumn{4}{|c|}{ Poisson $($ var. $=0.1)$} & \multicolumn{4}{|c|}{ Gaussian (var. = 0.1) } \\
\hline $\begin{array}{l}\text { Metrics } \\
\text { Types }\end{array}$ & 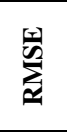 & 宏苞 & $\sum^{5}$ & $\sum$ & 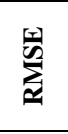 & 党党 & $\sum$ & $\sum_{\infty}$ & 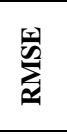 & 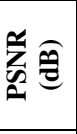 & $\sum^{5}$ & $\sum_{\mathscr{S}}$ & 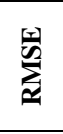 & $\sum_{\mathscr{\omega}}^{\mathscr{n}} \widehat{\theta}$ & $\sum^{5}$ & $\sum$ \\
\hline Median & 18.8 & 22.6 & 4.92 & 0.64 & 6.27 & 32.1 & 1.29 & 0.91 & 6.58 & 31.7 & 1.88 & 0.87 & 27.5 & 19.3 & 24.7 & 0.45 \\
\hline Wiener & 19.3 & 22.3 & 4.56 & 0.66 & 24.5 & 20.3 & 8.37 & 0.42 & 6.47 & 31.9 & 2.02 & 0.84 & 26.6 & 19.6 & 24.7 & 0.53 \\
\hline Mean & 14.4 & 24.9 & 3.85 & 0.67 & 19.6 & 22.2 & 6.98 & 0.47 & 7.33 & 30.8 & 1.93 & 0.86 & 27.2 & 19.4 & 24.9 & 0.49 \\
\hline H-Median & 17.3 & 23.3 & 4.01 & 0.65 & 8.13 & 29.9 & 1.35 & 0.88 & 8.07 & 29.9 & 1.89 & 0.85 & 27.5 & 19.3 & 24.9 & 0.46 \\
\hline Gaussian & 13.1 & 25.7 & 2.73 & 0.77 & 16.8 & 23.5 & 8.19 & 0.52 & 8.77 & 29.2 & 2.38 & 0.86 & 27.0 & 19.4 & 24.9 & 0.55 \\
\hline Bilateral & 21.5 & 21.4 & 5.11 & 0.59 & 41.5 & 15.7 & 9.78 & 0.21 & 13.7 & 25.3 & 4.08 & 0.76 & 29.1 & 18.8 & 25.5 & 0.48 \\
\hline NLM & 26.1 & 19.7 & 5.79 & 0.63 & 23.5 & 20.6 & 5.95 & 0.42 & 7.15 & 31.0 & 2.09 & 0.87 & 26.3 & 19.7 & 24.6 & 0.55 \\
\hline A-Diffusion & 31.1 & 18.2 & 8.32 & 0.58 & 49.7 & 14.1 & 9.85 & 0.16 & 5.92 & 32.6 & 1.80 & 0.88 & 30.3 & 18.4 & 25.5 & 0.36 \\
\hline
\end{tabular}

Fig. 6 shows a comparative visual analysis of clinical US image which is corrupted by speckle, salt and pepper, Poisson, and Gaussian noises, respectively, and denoised by several filtering algorithms, namely, median, Wiener, mean, hybrid median, Gaussian, bilateral, non-local means, and anisotropic diffusion, respectively. To find out the best filtering techniques for any particular noise through visual observation, some fundamental image quality indicators are considered, such as good image quality feature, edge preservation performance, less blurring, and overall clear visuality. Based on these particular indicators from Fig. 6(a-d), it can be observed that Gaussian filter, median filter, anisotropic diffusion filter, and non-local means filter give the enhanced performance for denoising speckle noise, salt and pepper noise, Poisson noise, and Gaussian noise from US image, respectively.

The quantitative data values of corresponding filtered images, as shown in Fig. 6, are enlisted in Table 1. The tabular data demonstrate the image quality assessment parameter values of eight different filtering techniques, which are applied to denoise US image against speckle noise, salt and pepper noise, Poisson noise, and Gaussian noise, respectively. In the case of speckle noise, among eight different filtering techniques, Gaussian filter gives the lowest RMSE value of 13.1 that indicates better fit, the highest PSNR value of $25.7 \mathrm{~dB}$ that specifies more amount of signal portion than noise, the lowest value of MAE of 2.73 that reveals the low amount of error, and the highest value of SSIM of 0.77 that specifies more similarity phenomenon between original US image and despeckled US image. But when the US image is corrupted by salt and pepper noise, the median filter gives the best results. For salt and pepper noise, the values of RMSE, PSNR, MAE, and SSIM are 6.27, 32.1, 1.29, and 0.91, respectively. In the case of Poisson noise, it is observed that the anisotropic diffusion filtering technique is better than others to denoise US image. 5.92, 32.6, 1.80, and 0.88 are the numerical values of RMSE, PSNR, MAE, and SSIM, respectively. Like the other three types of noises, Gaussian noise is also very severe in the case of US image. To remove Gaussian noise from US image, non-local means filtering technique provides the best results according to Table 1 data. The values of RMSE, PSNR, MAE, and SSIM are $26.3,19.7,24.6$, and 0.55 , respectively. 


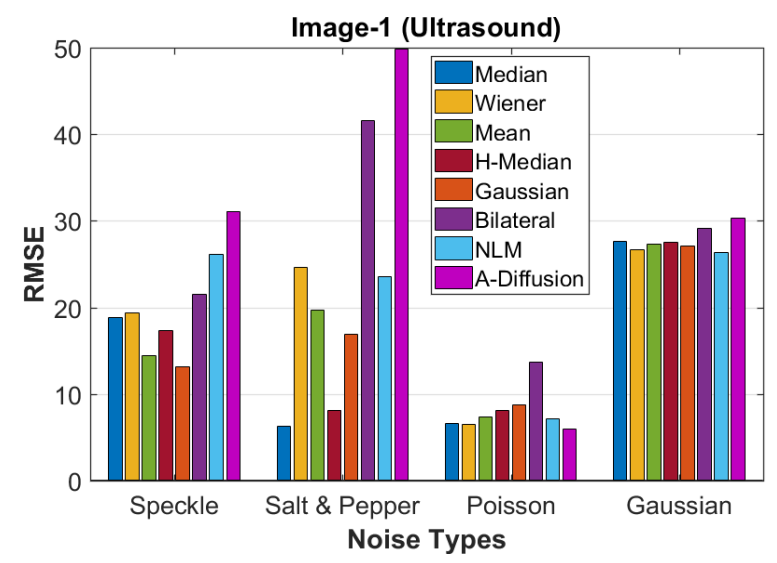

Fig. 7. RMSE values of different filtering techniques for speckle noise, salt and pepper noise, Poisson noise, and Gaussian noise in US image.

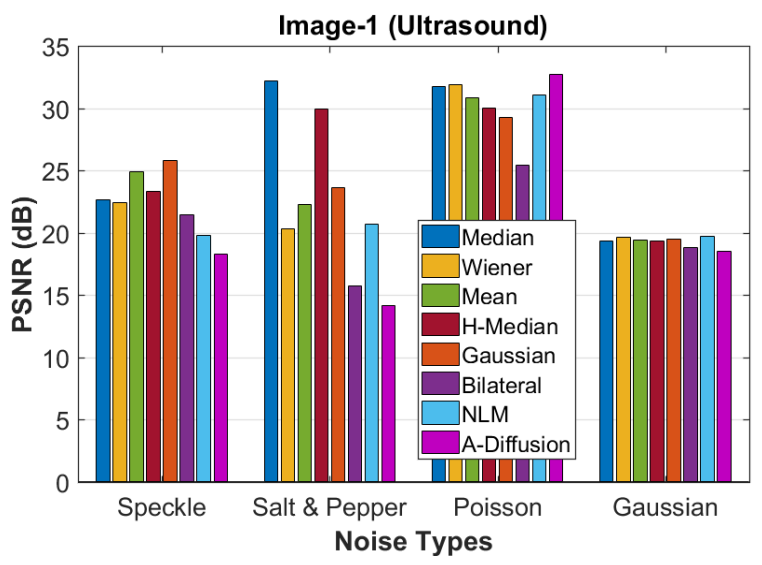

Fig. 8. PSNR values of different filtering techniques for speckle noise, salt and pepper noise, Poisson noise, and Gaussian noise in US image.

Here, Fig. 7 shows a bar chart that provides a comparative analysis of RMSE values of different filtering techniques for US image. In the case of speckle noise, the Gaussian filter provides the minimum RMES value, and the next lowest value is offered by mean filter. But for salt and pepper noise, the median filter and hybrid median filter show a comparatively lower value of RMSE. In the case of Poisson and Gaussian noises, the values of RMSE for most of the filtering techniques are very close to each other. Lowest RMSE values are provided by anisotropic diffusion, and non-local means filtering techniques for Poisson noise and Gaussian noise, respectively.

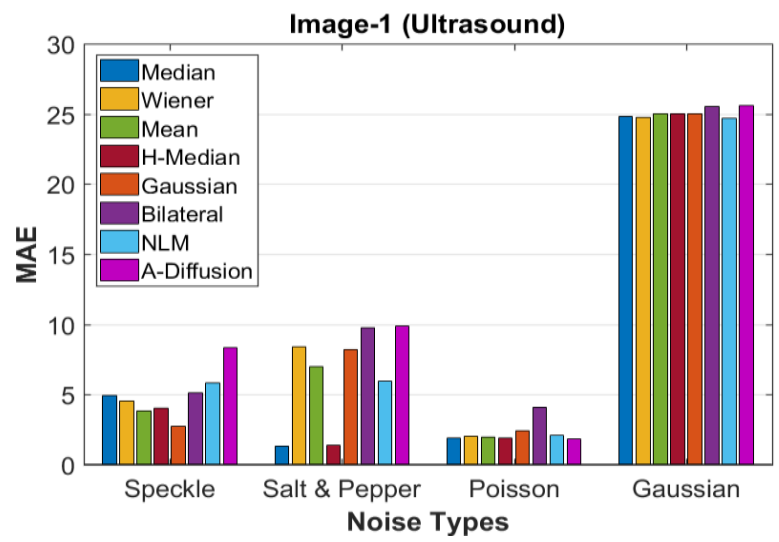

Fig. 9. MAE values of different filtering techniques for speckle noise, salt and pepper noise, Poisson noise, and Gaussian noise in US image. 


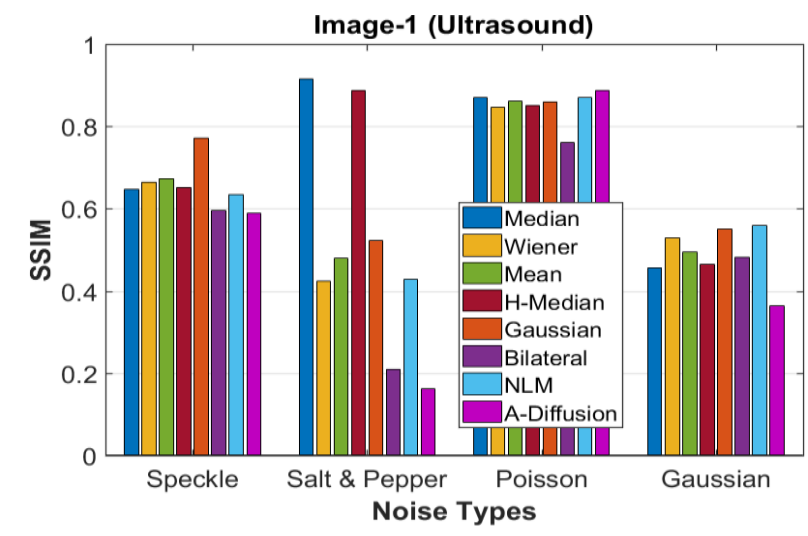

Fig. 10. SSIM values of different filtering techniques for speckle noise, salt and pepper noise, Poisson noise, and Gaussian noise in US image.

In Fig. 8, the PSNR values are shown graphically in $\mathrm{dB}$ form. Higher PSNR value is generally considered for any filtering techniques. From Fig. 8, it is clear to note that four different types of filtering techniques provide maximum PSNR values for four different noise types. In case of speckle noise, that filter is Gaussian, for salt and pepper noise that filter is median, for Poisson noise that filter is anisotropic diffusion, and in case of Gaussian noise that filter is non-local means. But for Gaussian noise, the PSNR values of other filtering methods are in close proximity to each other.

In the case of MAE, a low value indicates better performance. According to Fig. 9, Gaussian filter provides the lowest value of MAE in case of speckle noise. For salt and pepper noise, median, and hybrid median, both filters deliver low values of MAE. But in the case of Poisson and Gaussian filters, it is so close to separate the filtering techniques for providing low values of MAE.

From Fig. 10, the performance of different filtering techniques in terms of SSIM can be observed. The value of SSIM close to 1 is preferable. In the case of speckle noise, the value of SSIM is much higher for Gaussian filter than other types of filtering techniques. But in the case of salt and pepper noise, the median filter shows the highest value of SSIM. Like other IQA metrics, in the case of SSIM, anisotropic diffusion and non-local means filtering techniques provide better performance for Poisson and Gaussian noise, respectively.

Fig. 11 represents the CT image but in different conditions. The same CT image is corrupted by speckle noise, salt and pepper noise, Poisson noise, and Gaussian noise with intensity 10\%, respectively. After adding noises, several filtering methods, such as median, Wiener, mean, hybrid media, Gaussian, bilateral, non-local means, and anisotropic diffusion are applied to denoise the corrupted CT images. Fig. 11(a) expresses the despeckled CT images corresponding to different filtering methods. Similarly, Fig. 11(b-d) represent the denoised CT images, which are corrupted by salt and pepper noise, Poisson noise, and Gaussian noise, respectively. The careful observation indicates that the Gaussian filtered image, median filtered image, anisotropic diffusion filtered image, and non-local means filtered image are clearer and preserved about all the features of the original image in case of speckle noise, salt and pepper noise, Poisson noise, and Gaussian noise respectively.

Besides visual analysis to make a quantitative analysis based on IQA metrics, different tabular data, and graphical presentations are developed. Here, Table 2 shows the RMSE values of different filtering methods based on speckle noise, salt and pepper noise, Poisson noise, and Gaussian noise, respectively. In the case of speckle noise, the Gaussian filter provides a low RMSE value of 19.536, among other filtering techniques. But for salt and pepper noise, it is the median filter that gives a low RMSE value of 11.877. For rest of the two noises, i.e., Poisson and Gaussian noises, anisotropic diffusion and non-local means filtering methods deliver the lowest RMSE values of 7.094 and 26.440 respectively. The same scenarios are graphically presented in Fig. 12.

Based on PSNR, one of the most used IQA metrics, a similar type of results can be obtained from Table 3 and Fig. 13. Since the higher value of PSNR is desired, here, Gaussian filter, median filter, anisotropic diffusion filter, and nonlocal means filter provide higher PSNR values of 22.314, 26.637, 31.113, and 19.686 compared with others in case of speckle noise, salt and pepper noise, Poisson noise, and Gaussian noise respectively. Here, it is noted that in the case of Gaussian noise, the PSNR values of different filters are very close to each other.

The numerical values of MAE of different filtering techniques in the case of CT image are presented in Table 4, and its graphical illustration is shown in Fig. 14. MAE value of any filtered image indicates the amount of error that occurred during denoising the image, i.e., a low value of MAE is desired. Here, from Table 4 and Fig. 14 it can be observed that Gaussian filter, median filter, anisotropic diffusion filter, and non-local means filter ensure the lowest MAE values of 3.767, 1.800, 2.098, and 24.048 for speckle noise, salt and pepper noise, Poisson noise, and Gaussian noise respectively.

Unlike other IQA metrics, in case of SSIM, Gaussian filter, median filter, anisotropic diffusion filter, and non-local means filter provide the best results for speckle noise, salt and pepper noise, Poisson noise, and Gaussian noise, respectively. The highest values of SSIM of these particular filtering techniques corresponding to particular noise types are represented in Table 5 and Fig. 15. 
(a)
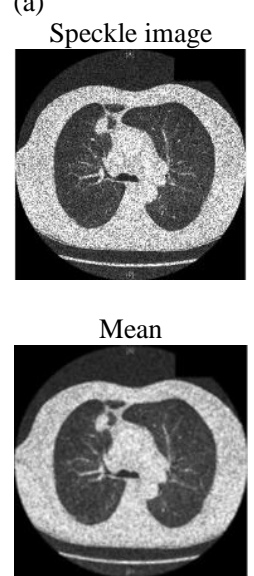

Bilateral

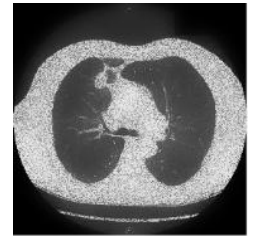

(c)

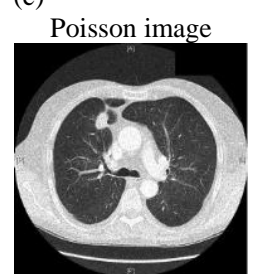

Mean

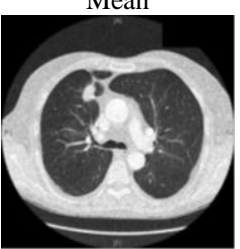

Bilateral

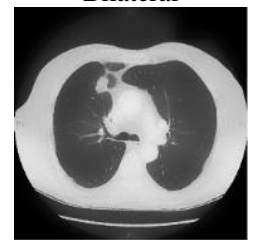

Median

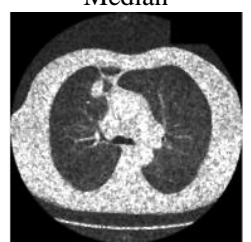

Hybrid Median

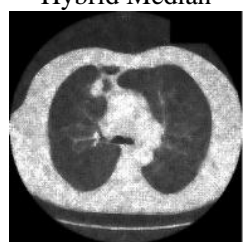

NLM

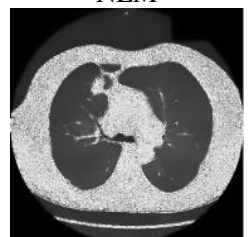

Median

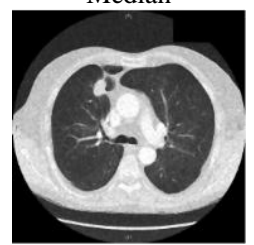

Hybrid Median

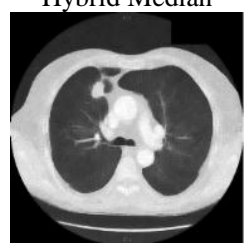

NLM

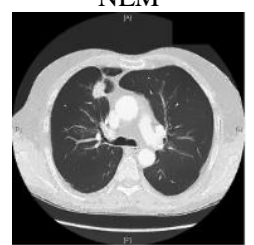

(b)

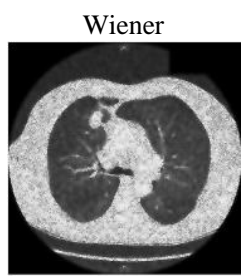

Gaussian
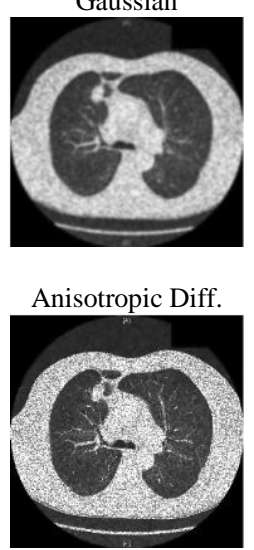

Wiener

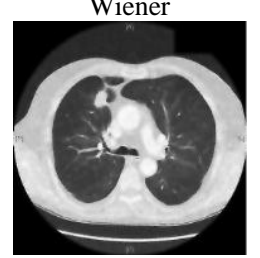

Gaussian

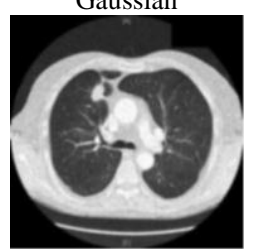

Anisotropic Diff.

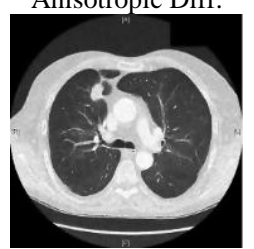

(d)
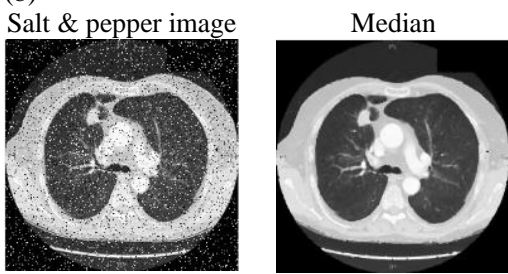

Hybrid Median

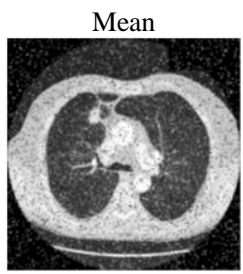

Bilateral
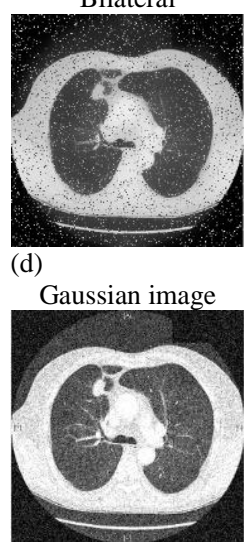

Mean

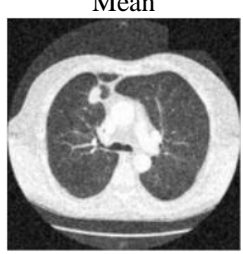

Bilateral

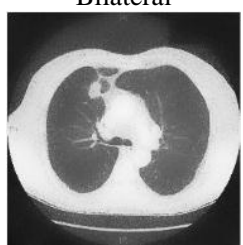

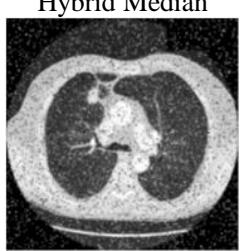

NLM

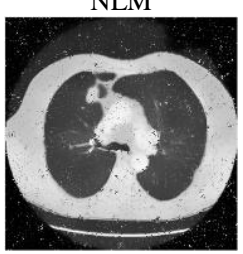

Median

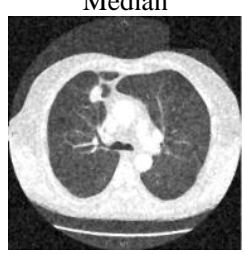

Hybrid Median

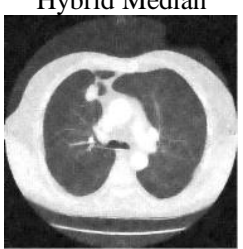

NLM

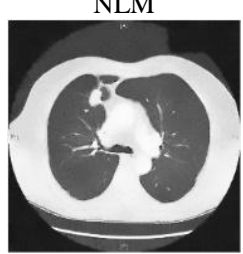

Wiener

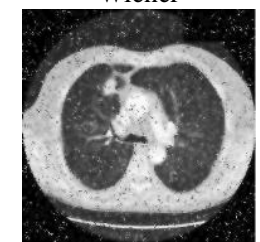

Gaussian

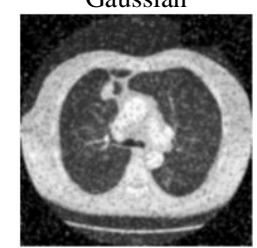

Anisotropic Diff.

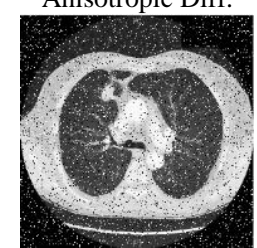

Wiener

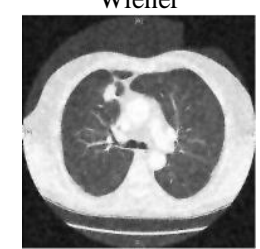

Gaussian
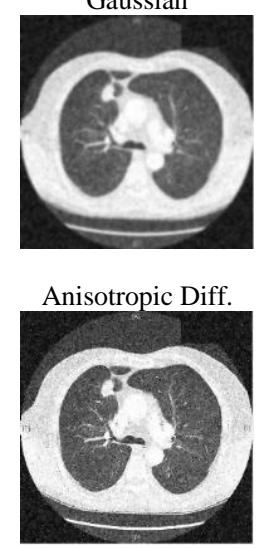

Fig. 11. Comparative visual quality analysis of CT images, denoised by different filtering techniques: Median, Wiener, Mean, Hybrid Median, Gaussian, Bilateral, Non-Local Means, and Anisotropic Diffusion. Here, (a) different filtered images corrupted by speckle noise $($ variance $=0.1)$; (b) different filtered images corrupted by salt $\&$ pepper noise (variance $=0.1$ ); (c) different filtered images corrupted by Poisson noise $($ variance $=0.1$ ); and (e) different filtered images corrupted by Gaussian noise (variance $=0.1$ ). 
Table 2. Quantitative RMSE values of different filtering techniques corresponding to speckle, salt and pepper, Poisson, and Gaussian noises with variance $=0.1$ in $2 \mathrm{D} 256 \times 256 \mathrm{CT}$ image.

\begin{tabular}{|c|c|c|c|c|}
\hline Metrics & \multicolumn{4}{|c|}{ RMSE } \\
\hline $\begin{array}{l}\begin{array}{r}\text { Noise } \\
\text { Types }\end{array} \\
\text { Filter } \\
\text { Types }\end{array}$ & 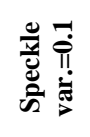 & 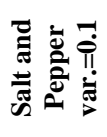 & 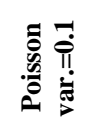 & 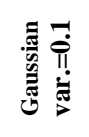 \\
\hline Median & 23.526 & 11.877 & 11.062 & 28.760 \\
\hline Wiener & 21.156 & 25.358 & 9.016 & 26.960 \\
\hline Mean & 19.778 & 22.840 & 13.486 & 28.647 \\
\hline H-Median & 22.566 & 12.632 & 11.847 & 28.900 \\
\hline Gaussian & 19.536 & 21.340 & 15.273 & 29.326 \\
\hline Bilateral & 27.598 & 41.461 & 14.115 & 29.204 \\
\hline NLM & 24.144 & 23.036 & 7.122 & 26.440 \\
\hline A-Diffusion & 33.641 & 49.943 & 7.094 & 29.738 \\
\hline
\end{tabular}

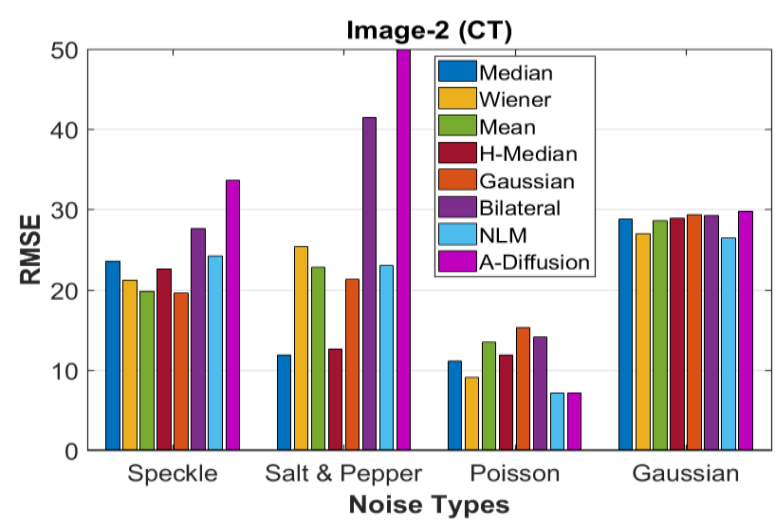

Fig. 12. RMSE values of different filtering techniques for speckle noise, salt and pepper noise, Poisson noise, and Gaussian noise in CT image.

Table 3. Quantitative PSNR values of different filtering techniques corresponding to speckle, salt and pepper, Poisson, and Gaussian noises with variance $=0.1$ in $2 \mathrm{D} 256 \times 256 \mathrm{CT}$ image.

\begin{tabular}{|c|c|c|c|c|}
\hline Metrics & \multicolumn{4}{|c|}{ PSNR } \\
\hline $\begin{array}{l}\begin{array}{r}\text { Noise } \\
\text { Types }\end{array} \\
\text { Filter } \\
\text { Types }\end{array}$ & 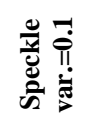 & 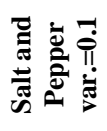 & 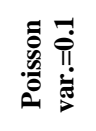 & 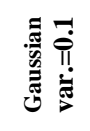 \\
\hline Median & 20.700 & 26.637 & 27.254 & 18.955 \\
\hline Wiener & 21.622 & 20.049 & 29.031 & 19.516 \\
\hline Mean & 22.207 & 20.957 & 25.533 & 18.989 \\
\hline H-Median & 21.062 & 26.101 & 26.659 & 18.913 \\
\hline Gaussian & 22.314 & 21.547 & 24.452 & 18.786 \\
\hline Bilateral & 19.313 & 15.778 & 25.137 & 18.822 \\
\hline NLM & 20.475 & 20.883 & 31.079 & 19.686 \\
\hline A-Diffusion & 17.593 & 14.161 & 31.113 & 18.664 \\
\hline
\end{tabular}

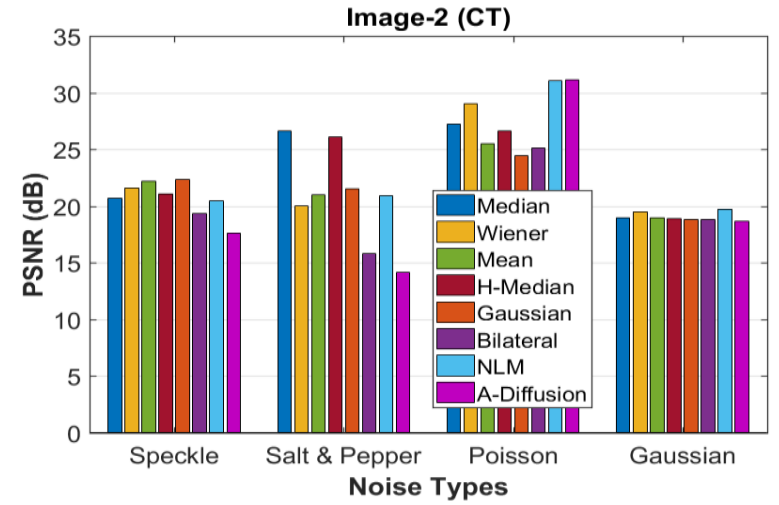

Fig. 13. PSNR values of different filtering techniques for speckle noise, salt and pepper noise, Poisson noise, and Gaussian noise in CT image. 
Table 4. Quantitative MAE values of different filtering techniques corresponding to speckle, salt and pepper, Poisson, and Gaussian noises with variance $=0.1$ in $2 \mathrm{D} 256 \times 256 \mathrm{CT}$ image.

\begin{tabular}{|c|c|c|c|c|}
\hline Metrics & \multicolumn{4}{|c|}{ MAE } \\
\hline $\begin{array}{l}\begin{array}{r}\text { Noise } \\
\text { Types }\end{array} \\
\text { Filter } \\
\text { Types }\end{array}$ & 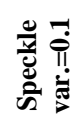 & 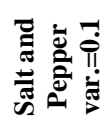 & 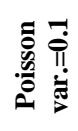 & 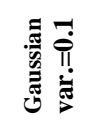 \\
\hline Median & 6.162 & 1.800 & 2.678 & 24.846 \\
\hline Wiener & 4.378 & 8.366 & 2.809 & 24.239 \\
\hline Mean & 4.113 & 6.964 & 3.123 & 24.226 \\
\hline H-Median & 4.897 & 2.148 & 2.364 & 25.358 \\
\hline Gaussian & 3.767 & 8.804 & 4.040 & 25.044 \\
\hline Bilateral & 5.707 & 9.175 & 4.325 & 24.717 \\
\hline NLM & 4.616 & 5.697 & 2.242 & 24.048 \\
\hline A-Diffusion & 7.869 & 9.545 & 2.098 & 24.877 \\
\hline
\end{tabular}

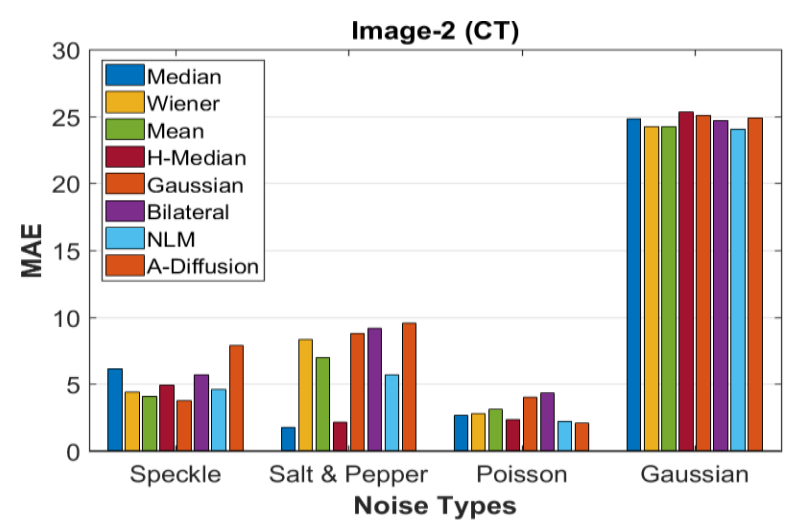

Fig. 14. MAE values of different filtering techniques for speckle noise, salt and pepper noise, Poisson noise, and Gaussian noise in CT image.

Table 5. Quantitative SSIM values of different filtering techniques corresponding to speckle, salt and pepper, Poisson, and Gaussian noises with variance $=0.1$ in $2 \mathrm{D} 256 \times 256 \mathrm{CT}$ image.

\begin{tabular}{|c|c|c|c|c|}
\hline Metrics & \multicolumn{4}{|c|}{ SSIM } \\
\hline $\begin{array}{l}\text { Noise } \\
\text { Fypes } \\
\text { Fypes } \\
\text { Types }\end{array}$ & 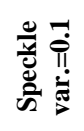 & 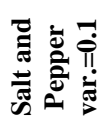 & 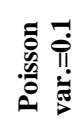 & 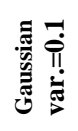 \\
\hline Median & 0.568 & 0.844 & 0.803 & 0.465 \\
\hline Wiener & 0.624 & 0.420 & 0.804 & 0.536 \\
\hline Mean & 0.643 & 0.459 & 0.753 & 0.496 \\
\hline H-Median & 0.583 & 0.836 & 0.802 & 0.482 \\
\hline Gaussian & 0.680 & 0.476 & 0.775 & 0.513 \\
\hline Bilateral & 0.529 & 0.254 & 0.718 & 0.497 \\
\hline NLM & 0.614 & 0.446 & 0.873 & 0.564 \\
\hline A-Diffusion & 0.596 & 0.233 & 0.879 & 0.433 \\
\hline
\end{tabular}

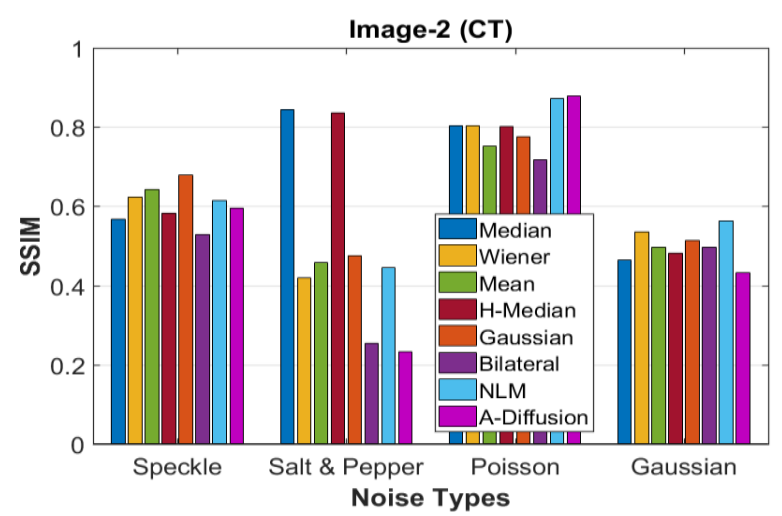

Fig. 15. SSIM values of different filtering techniques for speckle noise, salt and pepper noise, Poisson noise, and Gaussian noise in CT image. 
(a)

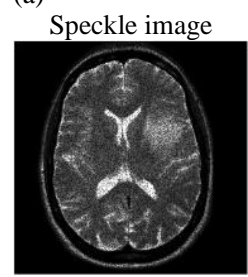

Mean

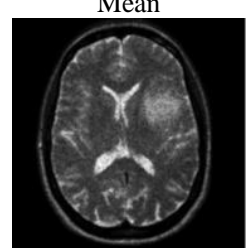

Bilateral

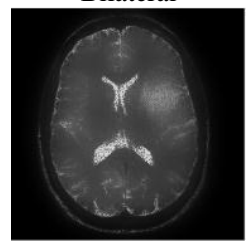

(c)

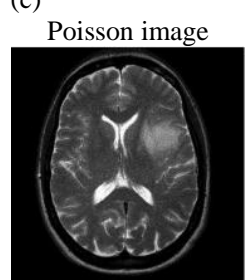

Mean

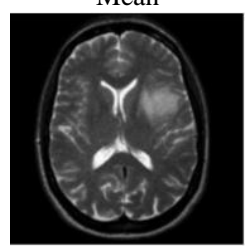

Bilateral

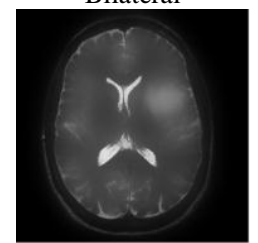

Median

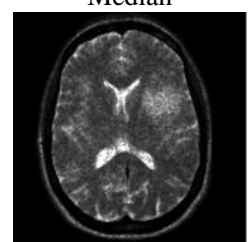

Hybrid Median

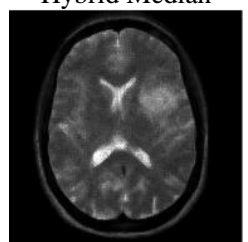

NLM

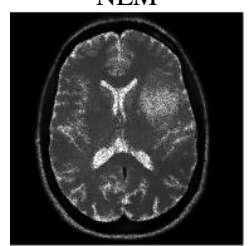

Median

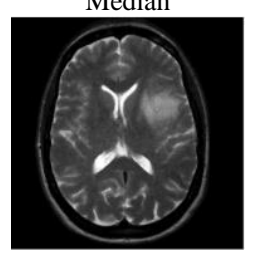

Hybrid Median

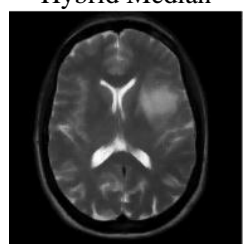

NLM

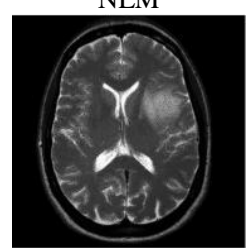

Wiener

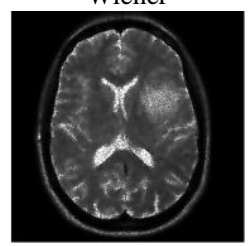

Gaussian

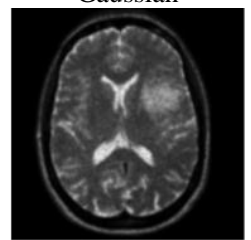

Anisotropic Diff.

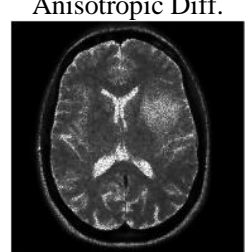

Wiener

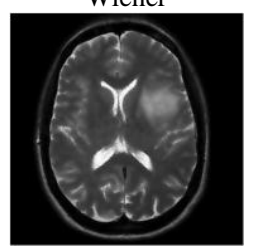

Gaussian

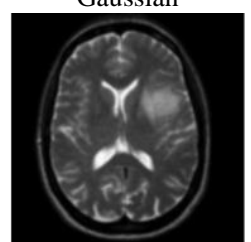

Anisotropic Diff.

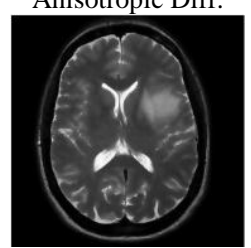

(b)

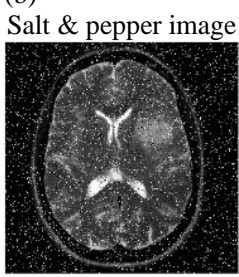

Mean

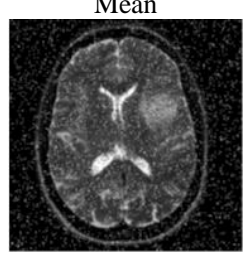

Bilateral

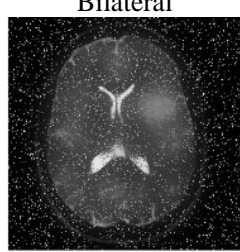

(d)

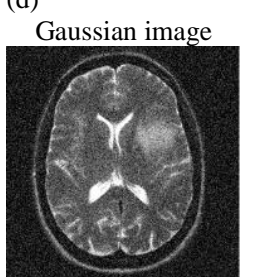

Mean

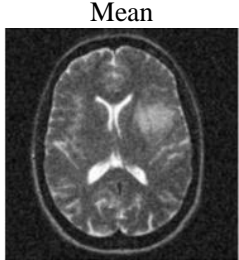

Bilateral

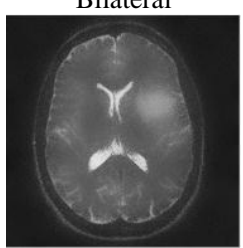

Median

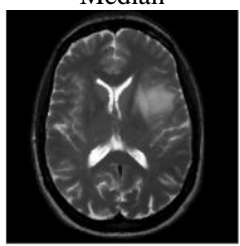

Hybrid Median

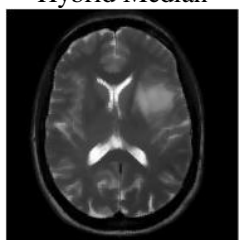

NLM

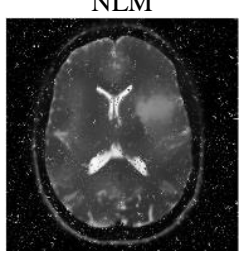

Median

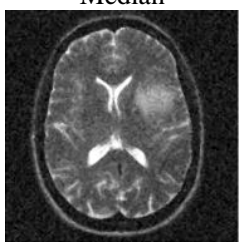

Hybrid Median

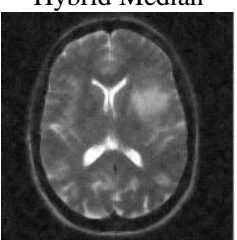

NLM

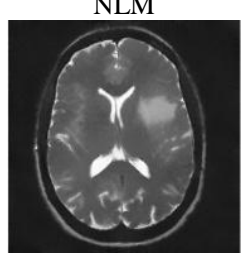

Wiener

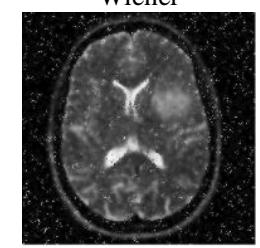

Gaussian

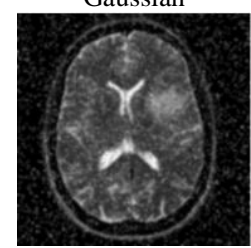

Anisotropic Diff

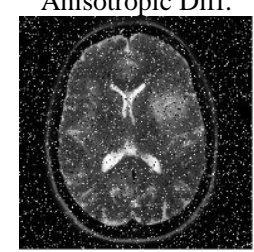

Wiener

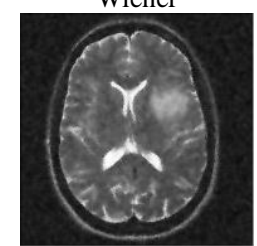

Gaussian

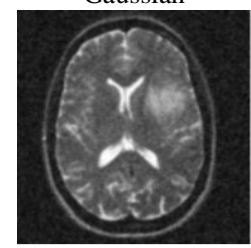

Anisotropic Diff.

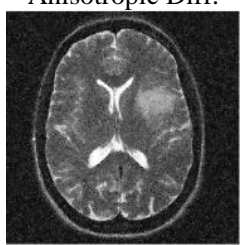

Fig. 16. Comparative visual quality analysis of MRI images, denoised by different filtering techniques: Median, Wiener, Mean, Hybrid Median, Gaussian, Bilateral, Non-Local Means, and Anisotropic Diffusion. Here, (a) different filtered images corrupted by speckle noise $($ variance $=0.1$ ); (b) different filtered images corrupted by salt \& pepper noise $($ variance $=0.1)$; $(\mathrm{c})$ different filtered images corrupted by Poisson noise $($ variance $=0.1)$; and (e) different filtered images corrupted by Gaussian noise (variance $=0.1$ ).

The same experiment is performed with MRI medical image, as shown in Fig. 16. From here, a relative ocular observation can be carried out on different filtered MRI images, which are corrupted by speckle noise, salt and pepper noise, Poisson noise, and Gaussian noise, respectively. Close observation in Fig. 16(a) reveals that among all images, Gaussian filtered image, mean filtered image, and bilateral filtered images are visually clearer. But the bilateral filtered image is more blurred than others. Again in terms of edge preservation performance and image quality, Gaussian filtered image is the best for speckle noise. Again in Fig. 16(b) the median and hybrid median filters almost remove entire salt and pepper noise from the MRI image. Among these two filters, the median filter performs better based on edge preservation quality. Similarly, Fig. 16(c) shows all the Poisson denoised MRI images, but in this case, visually, it is challenging to identify the best quality denoised image. Contrary, in the case of Gaussian noise, among all the filtered images, it is obvious to notice that non-local means filtered image has a good quality feature, edge preservation performance, less blurring, and overall precise visuality, as shown in Fig. 16(d). 


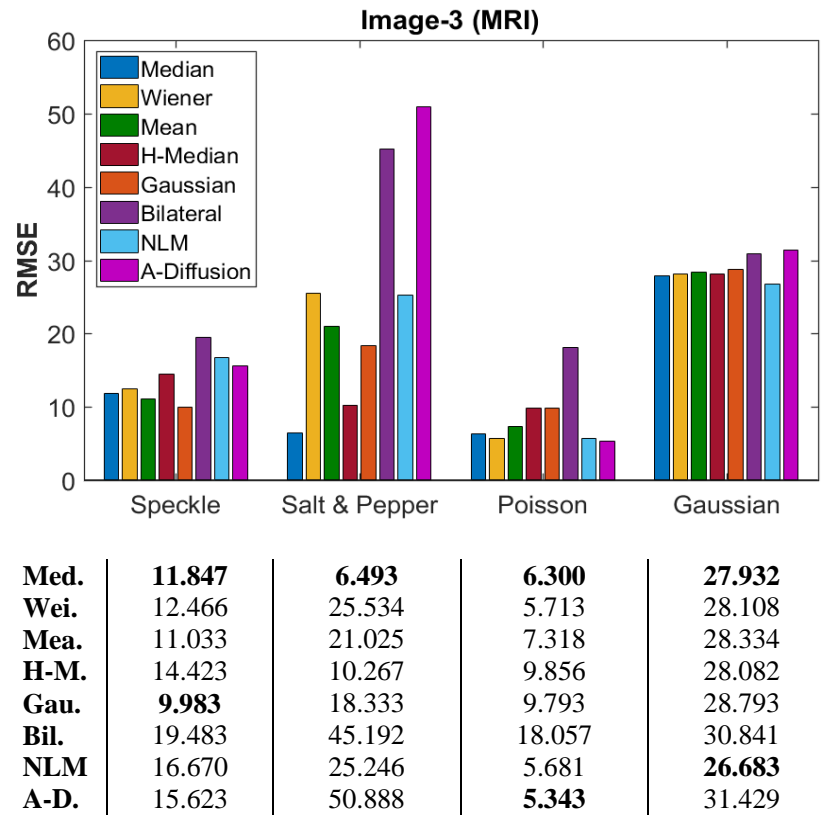

Fig. 17. RMSE values of different filtering techniques for speckle noise, salt and pepper noise, Poisson noise, and Gaussian noise in MRI image.

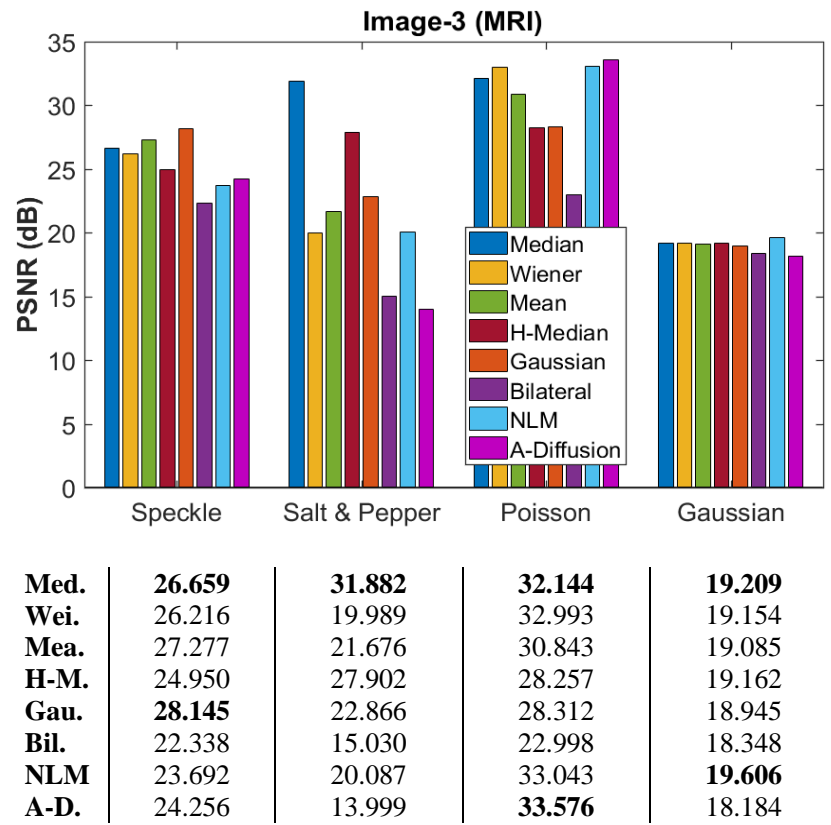

Fig. 18. PSNR values of different filtering techniques for speckle noise, salt and pepper noise, Poisson noise, and Gaussian noise in MRI image.

Here, Fig. 17 demonstrates the quantitative analysis of different filtering techniques for MRI images in terms of RMSE, one of the well-known image quality assessment metrics. In the case of speckle noise, Gaussian filter gives the lowest RMSE value of 9.983, whether, for salt and pepper noise this value is 18.333. Here, median filter delivers the lowest RMSE value of 6.493 for salt and pepper noise. Contrary, in case of Poisson and Gaussian noises, anisotropic diffusion and non-local means filtering methods provide a low RMSE value of 5.343 and 26.683 respectively. 


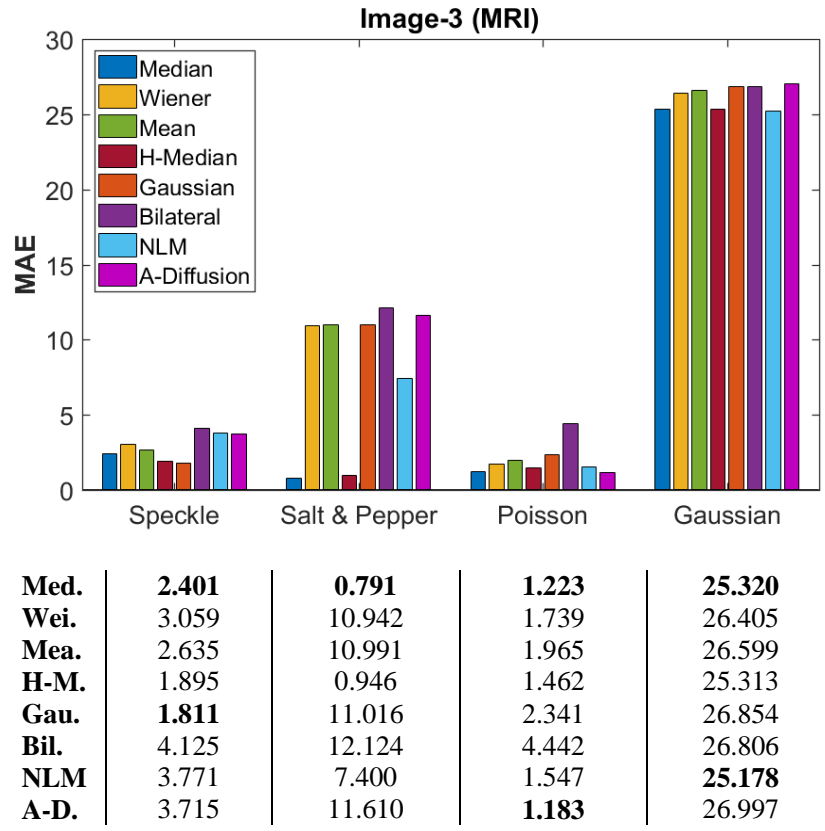

Fig. 19. MAE values of different filtering techniques for speckle noise, salt and pepper noise, Poisson noise, and Gaussian noise in MRI image.

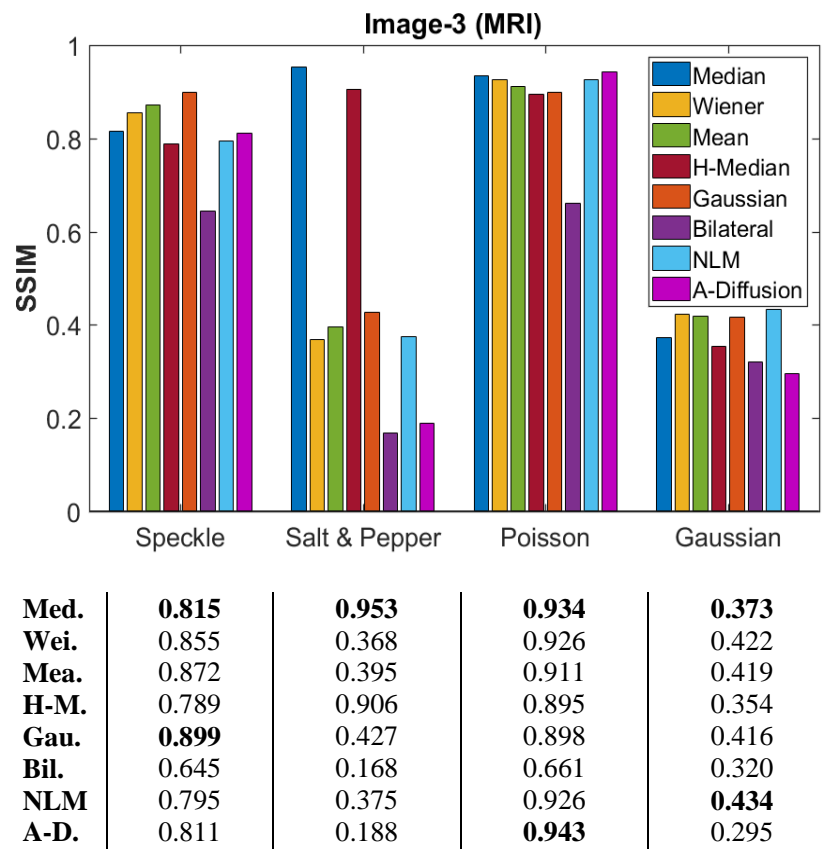

Fig. 20. SSIM values of different filtering techniques for speckle noise, salt and pepper noise, Poisson noise, and Gaussian noise in MRI image.

For MRI image, in the case of PSNR, another renowned image quality metric, a similar type of results can be found from Fig. 18. Here, more the value better is the performance. Based on that, the Gaussian filter, median filter, anisotropic diffusion filter, and non-local means filter ensure the highest PSNR values of $28.145 \mathrm{~dB}, 31.882 \mathrm{~dB}, 33.576$ $\mathrm{dB}$, and $19.606 \mathrm{~dB}$ for speckle noise, salt and pepper noise, Poisson noise, and Gaussian noise respectively.

In terms of MAE, the overall performance of all the filtering techniques for MRI images in the case of speckle, salt and pepper, Poisson, and Gaussian noises are presented gradually in Fig. 19. Here, both the graphical chart and numerical data make the comparative analysis easier. From Fig. 19 it can be observed that there are random variations of MAE values of different filtering methods in the case of four individual noise types. For particular speckle noise, among eight filtering techniques, Gaussian filter provides the minimum MAE value of 1.811 and the nearest value is 1.895 for hybrid median filter. In the case of salt and pepper noise, the best result is obtained from median filter with 0.791. For Poisson and Gaussian noises, minimum MAE values of 1.183 and 25.178 are gained from anisotropic diffusion, and non-local means filtering methods respectively.

Like RMSE, PSNR, and MAE, SSIM also provides the same type of results. Fig. 20 shows the SSIM values of different filtering techniques for speckle noise, salt and pepper noise, Poisson noise, and Gaussian noise in MRI image. 
With SSIM values of $0.899,0.953,0.943$, and 0.434 the Gaussian filter, median filter, anisotropic diffusion filter, and non-local means filter provide the superior performance in case of speckle noise, salt and pepper noise, Poisson noise, and Gaussian noise, respectively, though in case of Gaussian noise the SSIM value of non-local means filter is not up to the mark.

\section{Conclusion}

The existence of different types of noises in medical images is a common but undesirable phenomenon since it affects the quality of images very severely. So it is the crying need to get an effective filtering technique for removing a particular type of noise to ensure perfect diagnosis. In this paper, the performance of eight different denoising filtering techniques, namely, median, Wiener, mean, hybrid median, Gaussian, bilateral, non-local means, and anisotropic diffusion are studied based on RMSE, PSNR, MAE, and SSIM for four most hazardous noises, such as speckle, salt and pepper, Poisson, and Gaussian. Most importantly, all these noises and filtering methods are applied upon the three most widely used medical images, namely, US, CT, and MRI. Both statistical-quantitative and visual-qualitative performances are analyzed. The results reveal that the Gaussian filter is the best for despeckling US, CT, or MRI images. In the case of salt and pepper noise, the median filtering technique shows superior performance for all US, CT, and MRI images. Again, for removing Poisson noise from medical images (US, CT, MRI), the anisotropic diffusion filtering method is preferred. Finally, the non-local means appears as the finest filtering algorithm for eliminating Gaussian noise from US, CT, and MRI images. It should also be noted that we have performed the same analysis for other medical imaging techniques, for example, X-ray, OCT (Optical Coherence Tomography), and PET (Positron Emission Tomogra- phy). Most crucially, in every case, similar types of results are found.

\section{Acknowledgment} work.

The authors would like to thank the authority of Noakhali Science and Technology University for supporting this

\section{References}

[1] D. L. Donoho, "De-noising by soft-thresholding," IEEE Transactions on Information Theory, vol. 41, pp. 613-627, May 1995.

[2] R. D. Nowak, "Wavelet-based Rician noise removal for magnetic resonance imaging," IEEE Transactions on Image Processing, vol. 8, pp. 1408-1419, October 1999.

[3] M. Gupta, H. Taneja, and L. Chand, "Performance enhancement and analysis of filters in ultrasound image denoising," Procedia Computer Science, vol. 132, pp. 643-652, 2018.

[4] B. Goyall, A. Dogra1, S. Agrawal1, and B. S. Sohi, "Noise issues prevailing in various types of medical images," Biomedical \& Pharmacology Journal, vol. 11, pp. 1227-1237, September 2018.

[5] M. H. Ali, "MRI medical image denoising by fundamental filters," SCIREA Journal of Computer, vol. 2, pp. 12-26, 2017.

[6] J. W. Goodman, "Some fundamental properties of speckle," Journal of the Optical Society of America, vol. 66, pp. 1145-1150, November 1976.

[7] C. B. Burckhardt, "Speckle in ultrasound B-mode scans," IEEE Transactions on Sonics and Ultrasonics, vol. 25, pp. 1-6, January 1978.

[8] J. Sanches, J. Nascimento, and J. Marque, "Medical image noise reduction using the Sylvester-Lyapunov equation," IEEE Transactions on Image Processing, vol. 17, pp. 1522-1539, September 2008.

[9] W. R. Hedrick and D. L. Hykes, "Image and signal processing in diagnostic ultrasound imaging," Journal of Diagnostic Medical Sonography, vol. 5, pp. 231-239, September 1989.

[10] J. G. Abbott and F. L. Thurstone, “Acoustic speckle: Theory and experimental analysis," Ultrasonic Imaging, vol. 1, pp. 303324, October 1979.

[11] M. Diwakar and M. Kumar, "A review on CT image noise and its denoising," Biomedical Signal Processing and Control. Vol. 42, pp. 73-88, April 2018.

[12] M. Y. Jabarulla and H. N. Lee, "Computer aided diagnostic system for ultrasound liver images: A systematic review," Optik, vol. 140, pp. 1114-1126, July 2017.

[13] A. Lopes, R. Touzi, and E. Nezry, "Adaptive speckle filters and scene heterogeneity," IEEE Transactions on Geoscience and Remote Sensing, vol. 28, pp. 992-1000, November 1990.

[14] J. S. Lee, "Digital image enhancement and noise filtering by use of local statistics," IEEE Transactions on Pattern Analysis and Machine Intelligence, vol. 2, pp. 165-168, March 1980.

[15] D. T. Kuan, A. A. Sawchuk, T. C. Strand, and P. Chavel, "Adaptive noise smoothing filter for images with signal-dependent noise," IEEE Transactions on Pattern Analysis and Machine Intelligence, vol. 7, pp. 165-177, March 1985.

[16] R. C. Gonzalez and R. E. Woods, Digital Image Processing, $3^{\text {rd }}$ ed., Pearson Education Inc., London, UK, 2000.

[17] J. S. Goldstein, I. S. Read, and L. L. Scharf, "A multistage representation of the Wiener filter based on orthogonal projections," IEEE Transactions on Information Theory, vol. 44, pp. 2943-2959, November 1998.

[18] R. Maini and H. Aggarwal, "Performance evaluation of various speckle noise reduction filters on medical images," International Journal of Recent Trends in Engineering, vol. 2, pp. 22-25, December 2009.

[19] A. Achim, A. Bezerianos, and P. Tsakalides, "Novel Bayesian multiscale method for speckle removal in medical ultrasound images," IEEE Transactions on Medical Imaging, vol. 20, pp. 772-783, August 2001. 
[20] Z. J. Chen and C. H. Y. Chen, "Efficient statistical modeling of wavelet coefficients for image denoising," International Journal of Wavelets, Multiresolution and Information Processing, vol. 7, pp. 629-641, September 2009.

[21] L. Rubin, S. Osher, and E. Fatemi, "Nonlinear total variation based noise removal," Physica, vol. 60, pp. 259-268, November 1992.

[22] P. Perona and J. Malik, "Scale-space and edge detection using anisotropic diffusion," IEEE Transactions on Pattern Analysis and Machine Intelligence, vol. 12, pp. 629-639, July 1990.

[23] A. Buades, B. Coll, and J. M. Morel, "A review of image denoising algorithms, with a new one," Multiscale Model Simulation, vol. 4, pp. 490-530, January 2005.

[24] C. Tomasi, and R. Manduchi, "Bilateral filtering for gray and color images," Sixth International Conference on Computer Vision (IEEE Cat. No.98CH36271), Bombay, India, pp. 839-846, 1998.

[25] P. Hiremath, P. T. Akkasaligar, and S. Badiger, "Removal of Gaussian noise in despeckling medical ultrasound images," International Journal of Computer Science and Applications, vol. 1, pp. 25-35, July 2012.

[26] S. Wang, T. Z. Huang, X. L. Zhao, J. J. Mei, and J. Huang, "Speckle noise removal in ultrasound images by first and secondorder total variation," Springer, vol. 78, pp. 513-533, August 2018.

[27] M. Y. Jabarulla and H. N. Lee, "Speckle reduction on ultrasound liver images based on a sparse representation over a learned dictionary," Applied Sciences, vol. 8, pp. 903, May 2018.

[28] Z. Wang and E. P. Simoncelli, "Reduced-reference image quality assessment using a wavelet-domain natural image statistic model," Proc. SPIE 5666, Human Vision and Electronic Imaging X, pp. 149-159, March 2005.

[29] Z. Wang and H. R. Sheikh, "Image quality assessment: From error visibility to structural similarity," IEEE Transactions on Image Processing, vol. 13, pp. 600-612, April 2004.

[30] U. Sara, M. Akter, and M. S. Uddin, "Image quality assessment through FSIM, SSIM, MSE, and PSNR - A comparative study," Journal of Computer and Communications, vol. 7, pp. 8-18, January 2019.

[31] M. V. Sarode and P. R. Deshmukh, "Reduction of speckle noise and image enhancement of images using filtering technique," International Journal of Advancement in Technology, vol. 2, pp. 30-38, January 2011.

[32] R. H. Chan, C, W. Ho, and M. Nikolova, "Salt and pepper noise removal by median type noise detectors and detail-preserving regularization," IEEE Transactions on Image Processing, vol. 14, pp. 1479-1485, November 2005.

[33] D. M. Sugantharathnam and D. Manimegalai. "Removal of Poisson noise in medical images using FDCT integrated with Rudin-Osher_Fatemi model.” Biomedical Engineering: Applications, Basis and Communications, vol. 26, pp. 1450078, December 2014.

[34] A. K. Boyat and B. K. Joshi, "A review paper: Noise models in digital image processing," Signal \& Image Processing: An International Journal (SIPIJ), vol.6, April 2015.

[35] J. Jyoti and R. Shastri, "A study of speckle noise reduction filters," Signal \& Image Processing: An International Journal (SIPIJ), vol. 6, pp. 71-80, June 2015.

[36] S. Garg, R. Vijay, and S. Urooj, "Statistical approach to compare image denoising techniques in medical MR images," Procedia Computer Science, vol. 152, pp. 367-374, January 2019.

[37] B. Deepa and M. G. Sumithra, "Comparative analysis of noise removal techniques in MRI brain images," 2015 IEEE International Conference on Computational Intelligence and Computing Research (ICCIC), Madurai, pp. 1-4, 2015.

[38] Y. Yu and S. T. Acton, "Speckle reducing anisotropic diffusion," IEEE Transactions on Image Processing, vol. 11, pp. 12601270, November 2002.

\section{Authors' Profiles}

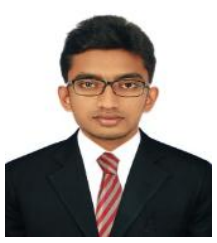

Shakil Mahmud Boby received a B.Sc. degree in Electrical and Electronic Engineering (EEE) from the Khulna University of Engineering and Technology (KUET), Khulna, Bangladesh, in 2018. Currently, he is serving Noakhali Science and Technology University (NSTU), Noakhali, Bangladesh, as a Faculty Member with the Department of Electrical and Electronic Engineering (EEE). He has been pursuing his M.Sc. degree in EEE from KUET, Khulna, Bangladesh. His research interests include Image Processing, Wireless Communications, 2D Electronic Devices and Nanotechnology.

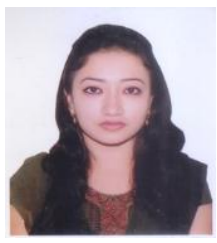

Shaela Sharmin received her B.Sc. degree in Electrical and Electronic Engineering (EEE) from the Ahsanullah University of Science and Technology (AUST), Dhaka, Bangladesh, in 2015, where she is currently a Faculty Member with the Department of Electrical and Electronic Engineering. She has been pursuing her M.Sc. degree in EEE from the same University. Her research interests include Digital Signal Processing, Wireless Communications, Cyber Security, and Smart Grid.

How to cite this paper: Shakil Mahmud Boby, Shaela Sharmin, " Medical Image Denoising Techniques against Hazardous Noises: An IQA Metrics Based Comparative Analysis", International Journal of Image, Graphics and Signal Processing(IJIGSP), Vol.13, No.2, pp. 25-43, 2021.DOI: 10.5815/ijigsp.2021.02.03 